\title{
Estimation of Cardiac Time Intervals From the Mechanical Activity of the Heart Using Machine Learning
}

\author{
Parastoo Dehkordi ${ }^{1}$, Kouhyar Tavakolian ${ }^{2}$, Te Zhao ${ }^{3}$, Vahid Zakeri ${ }^{3}$, and Farzad Khosrow-Khavar ${ }^{3}$ \\ ${ }^{1}$ Electrical and Computer Engineering Department, University of British Columbia, Vancouver, \\ Canada \\ ${ }^{2}$ Electrical Engineering Department, University of North Dakota, Grand Forks, USA \\ ${ }^{3}$ Heart Force Medical Inc., Vancouver, Canada
}

\begin{abstract}
Pre-ejection period (PEP) and total systolic time (TST) are hemodynamic indices which provide vital information about left ventricular performance. In this study, we suggested a method for estimating PEP and TST using the features extracted from the mechanical activity of the heart without finding the fiducial points associated with the opening and closure of heart valves. We recorded seismocardiogram (SCG) and gyrocardiogram $(G C G)$ signals from 50 healthy subjects using a 3-axial micro-electromechanical joint accelerometer-gyroscope sensor. For each axis of the SCG and GCG signals, 18 features were extracted per cardiac cycle. Several regression models were implemented using the features from multiple combinations of SCG and GCG and were validated against the measurements from tissue Doppler imaging (TDI) as the reference. The model that was fitted over the SCG z-axis features provided the most accurate estimates for PEP and TST intervals with the root mean square error of 9.5 and 17.2 millisecond, respectively. The estimation of cardiac time intervals investigated in this study broadens the potential of SCG and GCG in the monitoring of cardiovascular performance. As these technologies yield themselves to wearable applications, they could be used outside of hospital/clinical settings to detect abnormalities and malfunctions of the cardiovascular system.
\end{abstract}

\section{Introduction}

Cardiac time intervals are important hemodynamic indices that provide information about left ventricular performance. Two of the most important cardiac intervals are pre-ejection period (PEP) - defined as the time period between the onset of left ventricular depolarization (the onset of ECG Q) and the opening of the aortic valve - and total systolic time (TST) - defined as the time between ECG Q and the closure of the aortic valve [1]. Estimation of PEP and TST involves detecting the timing of the opening and closure of the aortic valve.

In clinical settings, the opening and closure of the aortic and mitral valves are commonly measured noninvasively using cardiac imaging modalities such as magnetic resonance imaging or echocardiography. These methods are time-consuming and require a trained sonographer to obtain accurate cardiac images. As such, there is a growing interest in finding simpler alternative techniques to measure cardiac intervals. Seismocardiography (SCG) and recently gyrocardiogram (GCG) have been suggested for this purpose.

SCG captures the chest acceleration along the $x^{-}, y-$, and $\mathrm{z}$-axis induced by the motion of myocardium. SCG is recorded using an accelerometer mounted on the lower part of the sternum. The fiducial points of a SCG cycle, labeled as $\mathrm{MC}, \mathrm{AO}, \mathrm{AC}$ and $\mathrm{MO}$, were suggested to correspond to mitral valve closure, aortic valve opening, aortic valve closure and mitral valve opening, respectively [2].

GCG has been recently introduced as a new noninvasive technology for measuring cardiac time intervals [3]. GCG captures the angular velocity of the chest caused by the heart's motion using a gyroscope placed on the sternum. The resulting angular velocity captured by a gyroscope sensor indicates the rate of angular displacement of the chest in terms of the speed of rotation around a specific axis. Three orthogonal uni-axial gyroscopes measure angular velocity along the $\mathrm{x}-, \mathrm{y}-$, and $\mathrm{z}$-axis.

The most challenging part of estimating PEP and TST from SCG and GCG is the delineation of the fiducial points. In this study, we investigated the estimation of PEP and TST from SCG and GCG recordings using the machine learning techniques that did not require the fiducial points to be annotated. We recorded 3-axial SCG and GCG from 50 healthy participants, developed a multivariate regression model to estimate PEP and TST using some characteristics from those recordings and then validated this method using tissue Doppler imaging as a reference. 


\section{MATERIAL AND METHODS}

\subsection{Data Set}

We recruited 50 male and female adults (19 - 85 years of age) with no known history of cardiovascular disease. The participants were initially scanned with echocardiography to detect any visible cardiac anomaly. Participants with any cardiac anomaly were excluded from further analysis. Participants were recruited following approval by the Office of Research Ethics at Simon Fraser University (2017s0241) and the obtaining of written informed consent.

To measure the heart's mechanical motion, a lownoise 3-axial micro-electro-mechanical (MEMS) joint accelerometer-gyroscope sensor (ASC GmbH, ASC IMU 7.002LN.0750, Germany) was used to record SCG and GCG. The sensor was mounted on the sternum close to the xiphoid while participants lay supine. Simultaneously, a two-lead ECG (iWorx Systems, Inc., IX-BIO8-SA, NH, USA) was recorded. All recordings were conducted using the iWorx data acquisition system (iWorx Systems, Inc., IX-416, NH, USA) sampled at $1 \mathrm{KHz}$ with 16-bit resolution.

In synchrony with the recording of the electromechanical activity of the heart, echocardiogram images were recorded using a GE Vivid q portable ultrasound machine with a cardiac GE M4S transducer (1.5-3.6 MHz) (GE Medical Systems, New York, US). For each subject, Tissue Doppler Imaging (TDI) modality was performed to record the apical views of at least 4 cardiac cycles.

\subsection{TDI Annotation}

The aortic valve openings and closures were manually annotated on the TDI cycles by an expert echocardiographer. Since valve opening/closure happens over a period of time from the initiation of the event until the completion point, instead of reporting a single time point, in this study, we reported a time span for each event (Figure 1a).

For each cycle, on the TDI images, $\mathrm{AVO}_{\text {min }}$ was marked at the exact moment before the annulus descended toward the apex and the $\mathrm{AVO}_{\max }$ was labelled as the point when the annulus started descending toward the apex. $\mathrm{AVO}_{t d i}$ was then marked as the midpoint between $\mathrm{AVO}_{\text {min }}$ and $\mathrm{AVO}_{\max }$.

At the end of systole, the myocardium reached the negative velocity. As the open aortic valve suddenly closed, there was a slight bounce, resulting in a shift from negative to positive velocity. $\mathrm{AVC}_{\min }$ and $\mathrm{AVC}_{\max }$ were marked respectively as the exact moments before and after the bounce. $\mathrm{AVC}_{t d i}$ were marked as the midpoint between $\mathrm{AVC}_{\min }$ and $\mathrm{AVC}_{\max }$.

\subsection{Feature Extraction}

After baseline removal using a moving median filter and smoothing with a Savitzky-Golay FIR filter (order 3, frame size 15 samples), all SCG and GCG signals were segmented into individual cycles using the $\mathrm{R}$ peaks detected manually from the synchronized ECG signal.

For each axis of the SCG and GCG signals, 18 features were extracted per cardiac cycle [4]. Twelve features, associated with systole, were extracted from the first part of each cycle, from ECG Q to ECG Q + $150 \mathrm{~ms}$ and six features, related to diastole, were extracted from the time interval between ECG Q + $250 \mathrm{~ms}$ and ECG Q + $450 \mathrm{~ms}$. The systolic features were as follows: the location of the first and second maxima, the amplitude of the first and second maxima, the height of the first maximum defined as the height between the point where the maximum begins and the peak of the maximum, the height of the second maximum defined as the height between the point where the maximum begins and the peak of the maximum, the slope of the first and second maxima, the location of the first and second minima, the amplitude of the first and second minima. The diastolic features were as follows: the location and amplitude of the maximum, the location of the first and second minima, the amplitude of the first and second minima.

\subsection{Multivariate Model Development and Validation}

The participants were randomly divided into training and test sets. In the training phase, the Least absolute shrinkage and selection operator (LASSO) method was employed to select the relevant features and to develop the final multivariate regression models [5]. The tuning parameter was adjusted using a 5-fold cross-validation.

To validate the models the leave-one-subject-out method was employed. In this method, the features of N-1 participants were assigned to the training set and were used to train the regression model. This model was then applied to predict the outcome for the only participant assigned to the test set. This procedure was repeated $\mathrm{N}$ times.

\subsection{Model Validation}

To validate the performance of the model, the root mean square error (RMSE) (ms) for PEP was defined as

$$
R M S E_{P E P}=\sqrt{\frac{1}{n} \sum_{i=1}^{n}\left(P E P_{\text {est }}-P E P_{\text {ref }}\right)}
$$

where $\mathrm{n}$ is the number of observations and $\mathrm{PEP}_{\text {ref }}$ and $\mathrm{PEP}_{\text {est }}$ are the reference $\left(\mathrm{Q}-\mathrm{AVO}_{\text {mid }}\right)$ and model esti- 


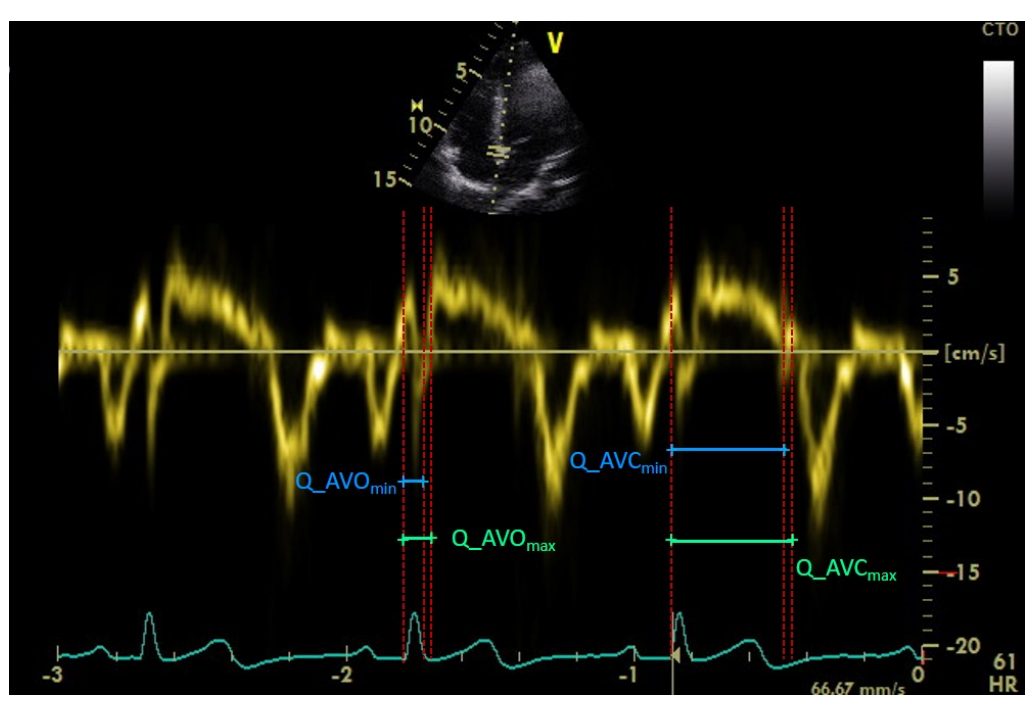

(a)

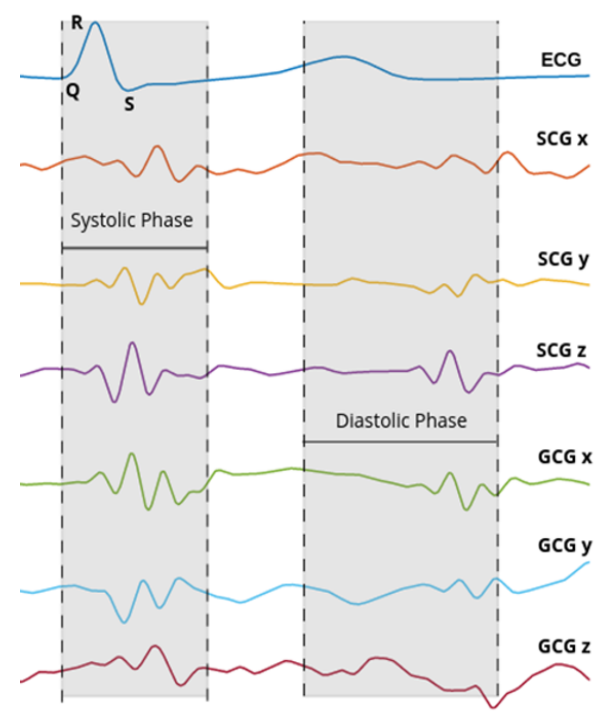

(b)

Figure 1: (a) TDI image. AVO and AVC stand for aortic valve opening and closure. Max and min subscripts represent the start and end of the echocardiographic intervals. (b) Recordings of ECG, 3-axial SCG and 3-axial GCG. The blurred rectangles show the systolic phase from ECG Q to ECG Q + $150 \mathrm{~ms}$ and diastolic phase from ECG Q + $250 \mathrm{~ms}$ to ECG Q $+450 \mathrm{~ms}$.

mates, respectively. $\mathrm{RMSE}_{T S T}$ was estimated between the reference TST $\left(\mathrm{Q}-\mathrm{AVC}_{\text {mid }}\right)$ and model estimates as well.

The agreement between the estimated PEP and TST time intervals and the reference TDI time intervals was assessed using the multiple-observation Bland-Altman method [6] and bias and two standard deviations 2SD were reported.

In addition, the percentage of cycles where the estimated PEP and TST timing intervals fell inside 5-millisecond margins of the corresponding echocardiography interval were reported.

\section{RESULT}

Eighteen features, for each axis of SCG and GCG, were extracted resulting in a total of 108 features per cycle for the combination of 3-axial SCG and 3-axial GCG. In total 220 cycles were analyzed.

Using all axes of SCG and GCG, RMSE REP $_{P}$ was measured as $9.57 \mathrm{~ms}$. More than $78 \%$ of $\mathrm{PEP}_{\text {est }}$ fell within the $\mathrm{AVO}_{\text {min }}$ and $\mathrm{AVO}_{\text {max }}$. The agreement between $\mathrm{PEP}_{\text {est }}$ and $\mathrm{PEP}_{t d i}$ was assessed by a Bland-Altman plot with a bias of $0.24 \mathrm{~ms}$ and 2SD of $19.1 \mathrm{~ms}$ (Table 1). The RMSE value for the PEP estimated from fiducial points of SCG $\mathrm{z}$-axis was calculated as $6.6 \mathrm{~ms}$ (Table 3 ).

The TST intervals estimated from the trained regression models were compared with the ones measured from TDI images (see Table 2). Results show that the SCG Z-axis signal provided the lowest RMSE of $17.2 \mathrm{~ms}$. The RMSE value for the TST estimated from fiducial points of SCG $\mathrm{z}$-axis was calculated as $7.5 \mathrm{~ms}$ (Table 3 ).

Table 1: Comparison of PEP estimated from different combinations of SCG and GCG axes with $\mathrm{PEP}_{t d i}$

\begin{tabular}{lcccc}
\hline \hline Axis & $\begin{array}{c}\text { RMSE } \\
(\mathrm{ms})\end{array}$ & $\begin{array}{c}\text { Bias } \\
(\mathrm{ms})\end{array}$ & $\begin{array}{c}\text { 2SD } \\
(\mathrm{ms})\end{array}$ & $\begin{array}{c}\text { \% in } \\
\text { TDI Range }\end{array}$ \\
\hline $\begin{array}{l}\text { Gx,Gy,Gz } \\
\text { Sx,Sy,Sz }\end{array}$ & 9.6 & 0.24 & 19.1 & 78 \\
\hline Gx,Gy,Gz & 10.3 & 0.14 & 20.5 & 71 \\
\hline Gx,Gy,Gz & 10.3 & 0.14 & 20.5 & 71 \\
\hline Sz & 9.5 & 0.18 & 19.1 & 77 \\
\hline Gx,Gy & 10.4 & 0 & 20.7 & 71 \\
\hline \hline
\end{tabular}

\section{DISCUSSION}

We proposed a novel method using multivariate regression models for the estimation of PEP and TST intervals based on the features extracted from different combinations of three-axial recordings of SCG and GCG signals. The results were validated using the measurements from TDI images. Estimation of PEP and TST using SCG and GCG signals is conventionally performed by annotation of the opening and closure of aortic valves in the recordings. 
Table 2: Comparison of TST estimated from different combinations of SCG and GCG axes with $\mathrm{TST}_{t d i}$

\begin{tabular}{lcccc}
\hline \hline Axis & $\begin{array}{c}\text { RMSE } \\
(\mathrm{ms})\end{array}$ & $\begin{array}{c}\text { Bias } \\
(\mathrm{ms})\end{array}$ & $\begin{array}{c}\text { 2SD } \\
(\mathrm{ms})\end{array}$ & $\begin{array}{c}\text { \% in } \\
\text { TDI Range }\end{array}$ \\
\hline Gx,Gy,Gz & 18.9 & 0.27 & 38.1 & 57 \\
Sx,Sy,Sz & & & & \\
\hline Gx,Gy,Gz & 17.4 & 0.07 & 35.4 & 61 \\
\hline Gx,Gy,Gz & 22.5 & 0.31 & 46.3 & 45 \\
\hline Sz & 17.2 & 0.21 & 34.9 & 62 \\
\hline Gx,Gy & 21.2 & 0.25 & 43.4 & 42 \\
\hline \hline
\end{tabular}

Table 3: Comparison of PEP and TST estimated using the SCG z-axes fiducial points with $\mathrm{PEP}_{t d i}$ and $\mathrm{TST}_{t d i}$

\begin{tabular}{lcccc}
\hline \hline $\begin{array}{l}\text { Time } \\
\text { Interval }\end{array}$ & $\begin{array}{c}\text { RMSE } \\
(\mathrm{ms})\end{array}$ & $\begin{array}{c}\text { Bias } \\
(\mathrm{ms})\end{array}$ & $\begin{array}{c}2 \text { SD } \\
(\mathrm{ms})\end{array}$ & $\begin{array}{c}\% \text { in } \\
\text { TDI Range }\end{array}$ \\
\hline PEP & 6.6 & 0.97 & 13.3 & 92 \\
\hline TST & 7.5 & -0.316 & 14.9 & 92 \\
\hline \hline
\end{tabular}

Yet due to the inherent complexity associated with signal morphologies that contain both inter- and intra-subject variability [7], finding fiducial points in SCG and GCG is extremely difficult. Therefore, the solution based on multivariate regression proposed in this paper can be considered as a promisingly powerful tool to estimate approximate cardiac time intervals without inducing implementation complexity.

In this study, we investigated the potential of using a combination of SCG and GCG recordings along different axes for improving the estimation of cardiac time intervals. However, the performance of the method based on a combination of 6 axes increased only slightly when compared with the performance calculated using SCG z-axes alone. The estimation of PEP and TST using only the features from SCG z-axes clearly had the smallest RMSE compared with using other combinations of 3-axial SCG and GCG signals (Table 1 and 2). Thus, even though we see the great potential of gyroscope recordings in monitoring the cardiovascular performance, the accuracy and the reliability of the multivariate regression model which used the GCG recordings do not increase significantly.

In a recent study based on the regression, the PEP was estimated using the features from the 3-axial SCG and GCG [4] and was compared with the same parameter estimated from ICG as a reference. Our results are comparable with the reported results of that study. However, in the current study, we have estimated the TST intervals in addition to the PEP estimates. Moreover, we used the PEP and TST parameters measured using TDI images.
PEP and TST estimated using the fiducial points annotated in the SCG signals provided significantly smaller RMSE compared to the error estimated for regression models (Table 3). It manifested that using the SCG fiducial points was still a better choice for estimating cardiac time when compared to estimating through the regression models. However, automatic annotation of SCG has its own challenges due to the beat to beat variation in the morphology of the signals.

The estimation of PEP and TST using SCG and GCG investigated in this study, offers an opportunity to assess cardiac contractility which, in addition to the analysis of the other features of these signals, broadens the potential of using SCG and GCG in the monitoring of cardiovascular performance. As these technologies yield themselves to wearable applications, they could be used outside of hos$\mathrm{pital} / \mathrm{clinical}$ settings to detect potential abnormalities and malfunctions of the cardiovascular system. Moreover, this technology can be used to monitor the improvement of cardiac performance in healthy individuals as a result of the adoption of a healthier and more active lifestyle.

\section{References}

[1] Boudoulas H. Systolic time intervals. Eur Heart J 1990; 11:93-104.

[2] Crow R, Hannan P, Jacobs D, Hedquist L, Salerno D. Relationship between seismocardiogram and echocardiogram for events in the cardiac cycle. American Journal of Noninvasive Cardiology 1994;8(1):39-46.

[3] Jafari Tadi M, Lehtonen E, Saraste A, Tuominen J, Koskinen J, Teräs M, Airaksinen J, Pänkäälä M, Koivisto T. Gyrocardiography: A new non-invasive monitoring method for the assessment of cardiac mechanics and the estimation of hemodynamic variables. Scientific Reports 2017;7(1):1-11.

[4] Shandhi M, Semiz B, Hersek S, Goller N, Ayazi F, O. I. Performance analysis of gyroscope and accelerometer sensors for seismocardiography-based wearable pre-ejection period estimation. IEEE J Biomed Health Inform 2019;1:1-10.

[5] James G, Witten D, Hastie T, Tibshirani R. An introduction to statistical learning. Springer, 2013.

[6] Bland J, Altman D. Statistical methods for assessing agreement between two methods of clinical measurement. Lancet 1986;1:307-310.

[7] Khosrow-Khavar, F Tavakolian K, Blaber A, Menon C. Automatic and robust delineation of the fiducial points of the seismocardiogram signal for non-invasive estimation of cardiac time intervals. IEEE Trans Biomed Eng 2016; 64(8):1701-10.

Address for correspondence:

Parastoo Dehkordi

1530-401 W Georgia St

Vancouver, BC V6B 5A1

parastoo.dehkordi@gmail.com 